\title{
EFFICACY OF THYMOQUINONE IN CHEMOPREVENTION AND TREATMENT OF ACRYLAMIDE-INDUCED STRIATED MUSCLE DEGENERATION OF RAT'S TONGUE (HISTOLOGICAL AND HISTOCHEMICAL STUDY)
}

\author{
Salah Gamal Salah Elsawy*, Kamal Abd-Elrhaman Kamal ${ }^{* *}$, Mohamed Gomaa Attia Zouair ${ }^{* * *}$
}

\begin{abstract}
Aim: To assess the effect of Thymoquinone as anew chemopreventive and treatment modality on acrylamide (ARC)-induced striated muscle degeneration of rat's tongue, the present study was performed. Material and methods: Forty adult male albino Sprague-Dawley rats were used as experimental animals and divided into four equal groups $(\mathrm{G}(\mathrm{s}))$ : 10 animals in each one for two successive periods ( 5 animals for 20 days: GA) and (the other 5 animals for 40 days: GB). The groups used were as follow: G1(A\&B) \{negative control group\}, G2(A\&B) \{positive control group: ARC treatment group\}, G3(A\&B)\{Thymoquinone chemoprevention group $\}$ : ARC + Thymoquinone, alternatively\} and G4(A\&B) \{Thymoquinone therapeutic group $\}$ In this group, animals were received ACR as mentioned in G2, followed by Thymoquinone $(20 \mathrm{mg} / \mathrm{kg})$ injection intraperitoneally once daily for 3 times as follow: first day, third day and the fifth day. After termination of the experiment, the tongue of the animals were examined then the animals were euthanized and the tongue were excised, fixed in $10 \%$ neutral buffered formalin, routinely processed and stained with hematoxylin and eosin (H\&E) stain as well as with PTAH stain in order to be examined microscopically and histomorphometrically. Results: Gross observation revealed that there was a variation in the areas of swelling, redness and bleeding that ranged from small patches in G3, moderate in G4 to large areas in G2. Histopathological findings demonstrated various alterations in the pattern of muscle fibers in the groups used throughout the experimental periods. These alterations included abnormal wavy course of the muscle fibers with splitting of the myofibers and fragmentation of the sarcoplasm. The histomorphometric results revealed that PTAH staining has variability in the area percentage throughout the groups used. There were significant differences between G1 and G2A, G1 and G3A, G1 and G4A, G2A and G4A, G3A and G4A. In conclusions: Realization of striated muscle damage in rat's tongue by ACR which becomes worse with prolonged duration. Moreover Thymoquinone has a significant protective effect when compared with its therapeutic effect on ACR-induced striated muscle injury of rat's tongue.
\end{abstract}

\section{INTRODUCTION}

The primary functions of skeletal musculature are locomotor activity, postural behavior, and breathing. However, skeletal muscle is susceptible to injury resulting from direct trauma or indirect causes ${ }^{(1)}$. A limitation to the comprehensive study of muscle injuries has been the lack of uniformity in their categorization and description. Reflecting this observation and to provide a framework from which to understand the historical progression of the classification and grading of muscle injuries, an interesting review has been published by Hamilton et al (2015) $)^{(2)}$. Matrices of some food industry are considered to significantly contribute to consumer dietary exposure to acrylamide $(\mathrm{ACR})^{(3-5)}$. Different ACR levels were detected in vending snacks and the differences 1-observed in the ACR content

\footnotetext{
* Demonstrator, Department of Oral and Dental Pathology, Faculty of Dental Medicine (Boys- Cairo), Al-Azhar University.

** Lecturer of Oral and Dental Pathology, Faculty of Dental Medicine (Boys- Cairo, Al-Azhar University.

*** Professor and Chairman of Oral and Dental Pathology Department, Faculty of Dental Medicine, (Boys-Cairo), Al-Azhar University.
} 
may result from several factors. The consumption of snacks from vending services contributes to the total human exposure to this toxic compound but, to date, only few countries have set limits for its presence in foods ${ }^{(6)}$. ACR is an odorless and colorless crystalline solid at room temperature. Its molecular formula is $\mathrm{CH} 2=\mathrm{CH}-\mathrm{CO}-\mathrm{NH} 2$ and molecular weight is 71.08 . ACR boiling point is $125^{\circ} \mathrm{C}(25 \mathrm{mmHg})$, and meltinge point is at $84.5^{\circ} \mathrm{C}$, and density is $1.27 \mathrm{~g} / \mathrm{ml} 25^{\circ} \mathrm{C}$ ). It is readily soluble in water and polar solvent such as acetone, methanol and ethanol. It is almost insoluble in nonpolar solvents such as carbon tetrachloride ${ }^{(7)}$. ACR is used in the personal care and grooming products, such as lotions, cosmetics and deodorants ${ }^{(8)}$. ACR is also, extensively used in industries and molecular laboratories for gel chromatography ${ }^{(8,9)}$. Moreover, ACR has been reported to be present in plant material like potatoes, carrots, radish, lettuce, Chinese cabbage, parsley, onions, spinach and rice paddy ${ }^{(10)}$ in sugar ${ }^{(11)}$ and olives ${ }^{(12)}$. Major health concerns are associated with ACR due to its various sources and methods of exposure as in drinking water, inhalation, skin absorption and occupational exposure ${ }^{(13)}$. Direct exposure to ACR may result from ingestion of high-carbohydrate foods prepared at high temperatures such as potato crisps, crackers and French fries ${ }^{(9)}$. Indirect exposure may result from residual traces of the monomer in food packaging where polyacrylamide is used as a binding agent ${ }^{(14)}$. ACR is formed in foods, if the heating frying is done in an oven, on a frying pan or by microwave heating, while no ACR has been detected in boiled food products ${ }^{(15)}$. It is generated from food components during heat treatment resulting from the reaction between the amino acid asparagines and reducing sugars ${ }^{(12)}$. ACR has been reported to be neurotoxic ${ }^{(16)}$, toxic to the reproductive system $^{(17)}$ and carcinogenic in experimental animals ${ }^{(18)}$. Al-Serwi et al $(2015)^{(19)}$ concluded that chronic exposure to ACR might lead to skeletal muscle damage in rat tongue which becomes worth with prolonged duration of exposure.

\section{Oxidative stress-mediated skeletal muscle degen- eration by ACR:}

The cytotoxic properties of ACR by affecting the cellular redox status may lead to generation of reactive oxygen species (ROS) causing cytotoxic and genotoxic effects ${ }^{(20)}$. It has been reported that the results of different animal models and patients indicate that elevated oxidative stress could be causative in inducing various signaling pathways which lead to muscle degeneration ${ }^{(21)}$. Oxidative stress is a loss of balance between the production of ROS during cellular metabolism and the mechanisms that clear these species to maintain cellular redox homeostasis. Increased oxidative stress has been associated with muscular dystrophy ${ }^{(22)}$. ROS are highly reactive oxygen containing molecules that are natural by-products of eukaryotic cellular metabolism ${ }^{(23)}$. Primary sources of ROS in cells are the membrane-bound nicotinamide adenine dinucleotide phosphate oxidase complex and the electron transport chain (ETC) in the mitochondria ${ }^{(24)}$. Production of ROS at the mitochondrial ETC is an unintended consequence of inefficiencies in the transfer of electrons between the complexes. The major sites of ROS leakage are believed to be complexes I and III, although other components of the ETC also have considerable contribution to the overall amount of ROS produced at the mitochondria ${ }^{(24)}$.

Muscle Regeneration: The maintenance of a working skeletal musculature is conferred by its remarkable ability to regenerate ${ }^{(1)}$. In mature mice and other mammals, a small population of quiescent myoblasts persists on the surface of the mature myofiber; these are called satellite cells and often referred to as myogenic stem cells. These quiescent satellite cells are activated in response to disturbances of myofiber homeostasis, and especially in response to necrosis of myofibers in order to form new muscle to replace the damaged segment. Satellite cells that 
give rise to myoblasts are considered essential in mammals for postnatal muscle growth and also for myogenesis and regeneration in response to muscle necrosis ${ }^{(25)}$. One possibility is that they may form new myofibers de novo in the interstitial space as has been reported to occur in some situations ${ }^{(26)}$. Some myoblasts start to differentiate and fuse together to form myotubes from about 3.5 days and myotube formation is almost completed by 7-10 days ${ }^{(27)}$. After this time, myotubes fuse with the resealed ends of the myofibers any damaged innervation is restored and the myofibers increase in size with regeneration probably being largely completed by 3 weeks after necrosis. This complex series of cellular and molecular events associated with classic regeneration is clearly a vastly different process to the relatively simple restoration of atrophic myofibers by hypertrophy: hence the need to be careful with language to clearly distinguish between these two situations ${ }^{(28)}$. Various biological approaches have targeted to improve skeletal muscle healing after injury. Several studies have shown that growth factors play a variety of roles during muscle regeneration ${ }^{(29,30)}$. The beneficial antioxidant effects of herbal extracts such as saffron, salvia leriifolia and Nigella sativa extracts in reducing or preventing muscle injury have been noticed in various studies ${ }^{(31,32)}$. Nigella sativa is commonly used as traditional medicine worldwide in the disease prevention due to less side-effect and affordable properties. Nigella sativa and its constituents are a rich source of antioxidant, an influential free radical scavenger and also modulator of various biological activities. Many components were characterized, but the major ones were thymoquinone (27.8\%-57.0\%). It has been reported that the content of thymoquinone in Nigella sativa is dependent on the country of origin; for example, the least content of thymoquinone $(1,274.6 \mathrm{mg} / \mathrm{kg})$ is observed in Sudan, whereas the highest is found in the specimens of Ethiopia $(3,098.5 \mathrm{mg} / \mathrm{kg})^{(33)}$.
The advances of various biological techniques combined with the identification and development of rodent models for muscular degeneration have contributed to the identification of molecular pathways involved in muscle regeneration ${ }^{(1)}$. In this regard, the ultimate goal of the current study is directed to investigate the effect of thymoquinone on ACR-induced striated muscle degeneration of rat's tongue.

\section{MATERIAL AND METHODS}

\section{I -Animal models:}

Forty adult male albino Sprague-Dawley rats weighting (200-250 g) were obtained from animal house, Cairo University (Egypt). The experimental animals were housed in standard cages with sawdust bedding under controlled environmental conditions of humidity $(30-40 \%)$, temperature $\left(20 \pm 2{ }^{\circ} \mathrm{C}\right)$, and light (12-h light/12-h dark). All experimental animals were maintained on standard diet, water and libitum. The experiment was conducted in the animal research unit of the Faculty of Pharmacy, Cairo, Boys, Al- Azhar University. The experiment was carried out according to the ethical and research committee protocol of the Faculty of Pharmacy, Cairo, Boys, Al-Azhar University. The procedures were conducted in accordance with the committee for the purpose of control and supervision on experiments on animals (CPCSEA Guidelines) ${ }^{(34)}$.

\section{II- Experimental design:}

After a week of adaptation, the animal group ${ }_{(\mathrm{s})}$ $\left(\mathrm{G}_{(\mathrm{s})}\right)$ were divided into four equal groups $(\mathrm{G}(\mathrm{s})): 10$ animals in each one for two successive periods (5 animals for 20 days: GA) and (the other 5 animals for 40 days: $\mathrm{GB})^{(19)}$ as follow: G1 nnegative control group\}: In this group, animals were fed with standard diet, without receiving any kind of treatment and considered as negative control group. G2 \{positive control group: ARC treatment group\}: In this group, animals were received $15 \mathrm{mg} / \mathrm{kg}$ 
body weight of ARC orally once daily by gastric tube ${ }^{(35)}$, and considered as positive control group. G3 \{Thymoquinone chemoprevention group\}: In this group, animals were received $15 \mathrm{mg} / \mathrm{kg}$ body weight of ACR orally on alternative day with Thymoquinone. Thymoquinone $(20 \mathrm{mg} / \mathrm{kg})$ was injected intraperitoneally $1 \mathrm{~h}$ prior to reperfusion after dissolved in distilled water ${ }^{(36)}$. This group was considered as chemoprevention group. G4 \{Thymoquinone therapeutic group: In this group, animals were received ACR as mentioned in G2, followed by Thymoquinone $(20 \mathrm{mg} / \mathrm{kg})$ injection intraperitoneally once daily for 3 times as follow: first day, third day and the fifth day and considered as therapeutic group ${ }^{(36)}$.

\section{III-Chemicals used:}

1-Acrylamide (99.9\% purity) was obtained from Sigma Aldrich chemical Company (Nasr cityCairo, Egypt). Its molecular formula is C3H5NO. The applied dose of $15 \mathrm{mg} / \mathrm{kg}$ was dissolved in distilled water and given orally by gastric tube ${ }^{(35)}$. 2-Thymoquinone was purchased from Sigma Aldrich chemical Company (Nasr city- Cairo, Egypt). The applied dose, was of (20 mg/kg) body weight, was administered intraperitoneally $1 \mathrm{~h}$ prior to reperfusion after dissolved in distilled water ${ }^{\left({ }^{(6)}\right.}$.

\section{IV- Sample collection:}

After each period of the investigation, the tongue's rat of the animals used were examined, then the animals were euthanized. After that, the rat's tongue was excised and fixed in $10 \%$ buffered neutral formalin solution for $24 \mathrm{~h}$. After fixation, the tongue was cut longitudinally into equal sections and trimmed with width of one $\mathrm{cm}$ average size.

\section{V- Preparation for histological examination:}

Tissue specimens were extracted, dissected and dehydrated in a series of alcohol solutions, cleaned in xylene, and infiltrated with paraffin wax, then embedded in paraffin blocks. Tissue sections were cut at $3 \mu \mathrm{m}$ thick, and routinely proceed for $\mathrm{H} \& \mathrm{E}$ staining for examination and recording the histological findings. Another tissue section was cut at $3 \mu \mathrm{m}$ for staining with Phosphotungestic acid haematoxylin stain (PTAH).

\section{VI- PTAH preparation ${ }^{(37)}$ :}

The PTAH kit was designed initially to stain neuroglia but at present it is used specially to differentiate between smooth muscular tissue and striated muscular tissue, since it stains isotropic bands of skeletal muscle's myofibrils. Moreover, it is one of the recommended methods to show fibrin.

\section{PTAH staining interpretation:}

The tissue sections were examined using light microscope to assess the prevalence of localization of PTAH within the tissues. In addition, image analysis computer system was used to assess area percentage of PTAH staining. The image analysis was performed using Leica QWIN V3 image analyzer computer system (Switzerland), the image analyzer consisted of a colored video camera, colored monitor and hard disc of Dell personal computer connected to the microscope and controlled by Leica Qwin V3 software. The image analyzer was first calibrated automatically to convert the measurement units (pixels) produced by the image analyzer program into actual micrometer units. This was done in the Oral and Dental Pathology Department, Faculty of Dental Medicine, Boys, Cairo, Al- Azhar University. The investigated parameters were assessed using area distribution of positive areas. It was measured in the form of area inside a standard measuring frame of area 11434.9 $\mu \mathrm{m}$ per 5 fields using a magnification (x200) by light microscopy transferred to the monitor. The selected fields had the most uniformly stained tissues were used for evaluation. These areas were masked by a blue binary color using the computer system for measurement. Mean values were obtained for the whole specimens in each group. The collected data 
were recorded and statistically analyzed. One way analysis of variance (ANOVA) test will be used for comparison between the groups. In addition, Tukey's post hoc test will be performed when ANOVA test reveal significant difference.

\section{RESULTS}

The Gross observation results of tongue mucosa in the various groups used, throughout the experimental periods, showed various changes compared to G1( $\underline{\text { Plate. } 1)}$.

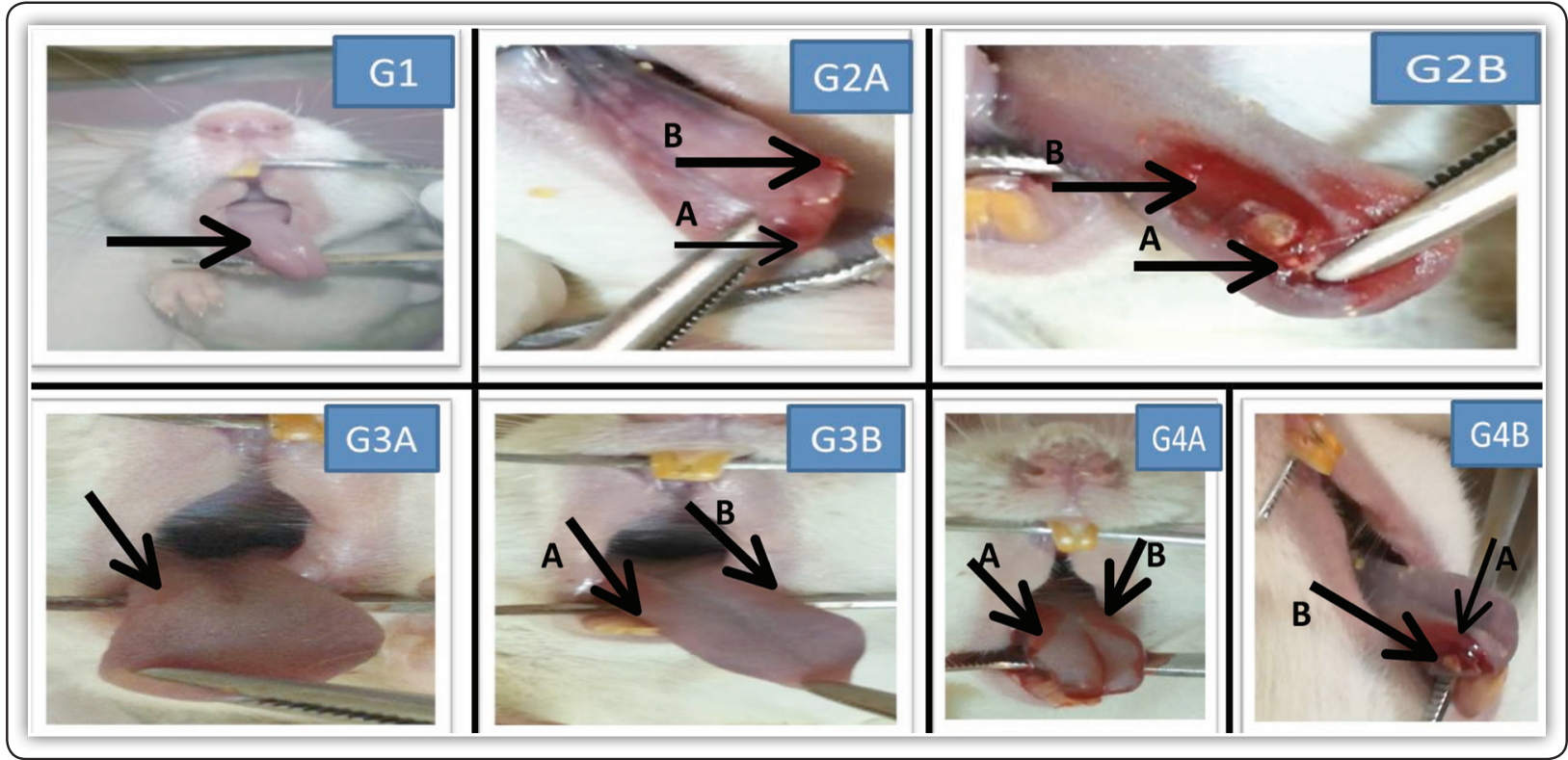

Plate.1: Gross observation of tongue mucosa in G1 showing normal appearance (arrow). Tongue mucosa in G2A showing small swelling (arrow A), redness and bleeding (arrow B). Tongue mucosa in G2B showing multiple swelling (arrow A), redness and bleeding (arrow B). Tongue mucosa in G3A showing limited localized redness (arrow). Tongue mucosa in G3B showing swelling (arrow A) and areas of redness (arrow B). Tongue mucosa in G4A showing swelling (arrow A) and areas of redness (arrow B). Tongue mucosa in G4B showing multiple swelling (arrow A), and bleeding (arrow B).

\section{Histopathological and histochemical results:}

Histological section of the dorsal surface of the tongue in G1, using $\mathrm{H} \& \mathrm{E}$ stain, showed that the overlying stratified epithelium is composed of multiple layers, with a well-formed rete ridges. The epithelium was seen to be variable from to fully keratinized epithelium overlying the filiform papillae more anteriorly (the anterior two-third) to parakeratinized stratified squamous epithelium posteriorly (the posterior third). Subepithelial lamina propria showed connective tissue fibers without inflammatory cells, the underlying connective tissue is composed of bundles and fascicles of striated muscle fibers. The later were seen to be arranged in different directions separated by perimysial connective tissue. The muscle fibers appeared parallel, long, cylindrical and non-branching with acidophilic crossly striated sarcoplasm. The nuclei were vesicular, elongated and peripheral in position under the sarcolemma. The fibers were connected together by connective tissues endomysium that showed flat nuclei of fibroblasts (Plate 2, Fig 1). PTAH stain exhibited subepithelial collagen fibers with brownish color. A closely packed uniform pattern of parallel bundles of striated muscle fibers 
with blue color that had regular striations was seen. The nuclei appeared dark blue with peripheral location in the cells of the muscle fibers. Elastic fibers appeared pale brownish red between the muscles (Plate 2, Fig 2). The histomorphometric analysis recorded that the mean of surface area of PTAH staining throughout the muscle fibers is $70.34 \%$. Histological section of the dorsal surface of the tongue in G2A showed that the overlying keratinized stratified squamous epithelium is composed of a well-formed keratinized papillae and subepithelial lamina propria infiltrated by inflammatory cells. Irregular pattern of muscle fibers with dense pyknotic nuclei as well as splitting of the myofibers and fragmentation of the sarcoplasm were seen (Plate 2, Fig.3). PTAH stain exhibited subepithelial collagen fibers with brownish color and irregular pattern of muscle fibers with variability in stainability. Muscle fibers with irregular striations and spaces in-between were seen. Limited areas of elastic fibers and a separation of muscle fibers were also seen (Plate 2, Fig.4). The histomorphometric analysis recorded that the mean of surface area of PTAH staining throughout the muscle fibers is $(40.99 \%)$. Histological section of the dorsal surface of the tongue in G2B showed hyperplastic keratinized stratified squamous epithelium, a wellformed rete ridges are more elongated and closer to each other, with few interpapillary surfaces. Subepithelial lamina propria infiltrated by inflammatory cells (lymphocytes, plasma cells and macrophages) around blood vessels was seen. The muscle fibers revealed irregular pattern, variable sizes and wavy course, in addition to multiple spaces in-between and fragmentation of the sarcoplasm (Plate 2, Fig.5). PTAH stain exhibited subepithelial collagen fibers with brownish color and wide irregular pattern and separation of muscle fibers. The latter showed few nuclei, irregular striations and splitting in addition to multiple spaces in-between and limited areas of elastic fibers (Plate 2, Fig.6).
The histomorphometric analysis recorded that the mean of surface area of PTAH staining throughout the muscle fibers is $(27.14 \%)$. Histological section of the dorsal surface of the tongue in G3A showed keratinized stratified irregular acanthotic rete ridges, subepithelial lamina propria infiltrated by few numbers' inflammatory cells, and blood vessels engorged RBCs. Some muscle fibers still were seen to have wavy course while others showed splitting with limited areas of spaces in-between (Plate 3, Fig.7). PTAH stain exhibited subepithelial collagen fibers with brownish color, almost regular and uniform pattern of muscle fibers with deeply stained peripherally located nuclei. Limited narrow spaces between the muscle fibers and areas of purplish elastic fibers between the muscle were seen (Plate 3, Fig.8). The histomorphometric analysis recorded that the mean of surface area of PTAH staining throughout the muscle fibers is (47.54). Histological section of the dorsal surface of the tongue in G3B showed keratinized stratified squamous epithelium, regular acanthotic rete ridges and subepithelial lamina propria infiltrated by inflammatory cells. Irregular pattern of wavy course muscle fibers with variable sized spaces in between was seen. (Plate 3, Fig.9). PTAH stain exhibited brownish subepithelial connective tissue fibers, irregular pattern of muscle fibers and limited areas with variable sized narrow spaces between the muscle (Plate 3, Fig.10). The histomorphometric analysis recorded that the mean of surface area of PTAH staining throughout the muscle fibers is $(31.23 \%)$ with small and pale basophilic fibers. Histological section of the dorsal surface of the tongue in G4A showing broad acanthotic rete ridges, subepithelial lamina propria infiltrated by inflammatory cells, and blood vessels engorged RBCs. Abnormal wavy course of muscle fibers with limited areas of the spaces in-between was observed (Plate 3, Fig.11). PTAH stain exhibited brownish subepithelial connective tissue fibers, irregular pattern of muscle 
fibers and variable-sized spaces in-between. Limited areas of variable sized narrow spaces between the muscle fibers were seen (Plate 3, Fig.12). The histomorphometric analysis recorded that the mean of surface area of PTAH staining throughout the muscle fibers is $(28.85 \%)$. Histological section of the dorsal surface of the tongue in G4B showed acanthotic rete ridges and subepithelial lamina propria infiltrated by inflammatory cells. An irregular wavy course in addition to splitting and fragmentation of muscle fibers with variable sized spaces in-between were seen (Plate 3, Fig.13). PTAH stain exhibited brownish subepithelial connective tissue fibers, irregular pattern of muscle fibers and variable-sized spaces between the muscle fibers with splitting of the myofibers and fragmentation of the sarcoplasm. Limited areas of variable sized wide spaces between the muscle fibers with small and pale basophilic fibers were observed (Plate 3, Fig.14). The histomorphometric analysis recorded that the mean of surface area of PTAH staining throughout the muscle fibers is $(28.33 \%)$.

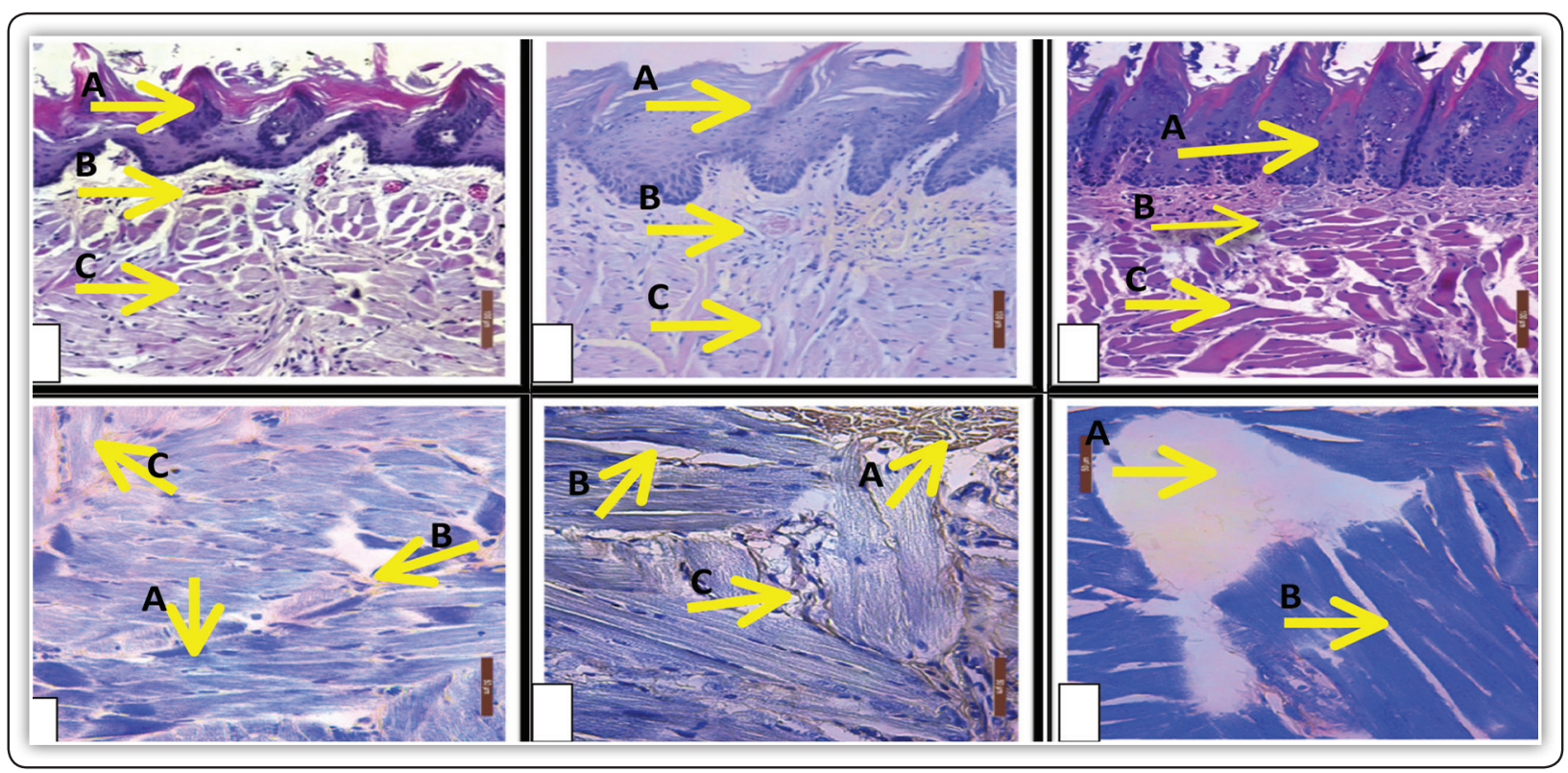

Plate.2: Fig.1: H\&E stain in tongue tissue section in G1 showing stratified squamous epithelium (arrow A), subepithelial connective tissue fibers without inflammatory cells (arrow B), fascicles of muscle fibers with multiple nuclei (arrow C). Fig.2: PTAH stain in tongue tissue section in G1 showing closely packed uniform pattern of parallel bundles of striated muscle fibers (bluish color)(arrow A) with regular striations(arrow B), deeply stained peripherally located nuclei and elastic fibers appeared with delicate linear brownish red staining (arrow C). Fig.3: H\&E stain in G2A showing keratinized stratified squamous epithelium with acanthotic rete ridges (arrow A), subepithelial mesenchymal tissue infiltrated by inflammatory cells (arrow B), irregular pattern of muscle fibers with splitting of the myofibers and fragmentation of the sarcoplasm (arrow C). Fig.4: PTAH stain showing limited areas of elastic fibers (linear, brownish) (arrow A) and a separation of muscle fibers (pale bluish color) (arrow B) with deeply stained peripherally located nuclei (arrow C). Fig.5: H\&E stain in G2B showing hyperplastic keratinized stratified squamous epithelium(arrow A), subepithelial mesenchymal tissue infiltrated by inflammatory cells(arrow B) and irregular pattern of muscle fibers with variable sized multiple spaces (arrow C). Fig.6: PTAH showing wide irregular (arrow A) as well as fusiform spaces between the muscle fibers (arrow B) with almost an absence of nuclei and limited areas of elastic fibers. 


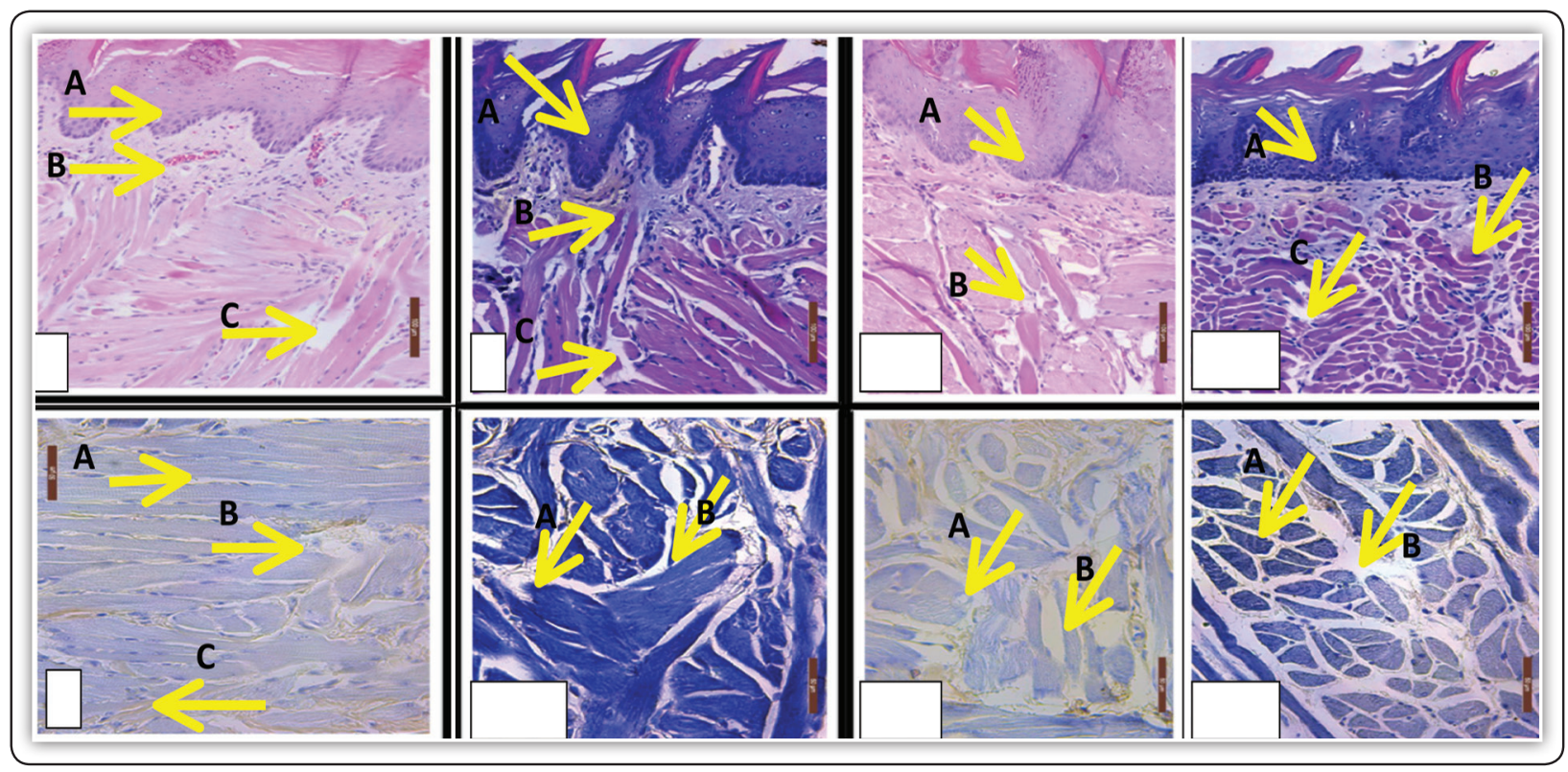

Plate.3: Fig.7: H\&E stain in G3A showing keratinized stratified irregular acanthotic rete ridges (arrow A), subepithelial mesenchymal tissue infiltrated by inflammatory cells, and blood vessels engorged RBCs(arrow B) and abnormal wavy course of muscle fibers with limited areas of spaces in between (arrow C). Fig.8: PTAH stain showing uniform pattern of muscle fibers(arrow A), areas of elastic fibers (brownish color)(arrow B), and limited narrow spaces between muscle fibers with deeply stained peripherally located nuclei (deep blue) and limited spaces between the muscle fibers (arrow C). Fig.9: H\&E stain in G3B showing keratinized stratified regular acanthotic rete ridges(arrow A), subepithelial mesenchymal tissue infiltrated by inflammatory cells (arrow B) and irregular pattern of wavy course muscle fibers with variable spaces in between (arrow C). Fig.10: PTAH stain showing irregular pattern of muscle fibers (arrow A), limited areas of collagen fibers and variable sized narrow spaces between the muscle fibers (arrow B). Fig.11: H\&E stain in G4A showing keratinized stratified regular broad acanthotic rete ridges; subepithelial mesenchymal tissue infiltrated by inflammatory cells, blood vessels engorged RBCs (arrow A) and abnormal wavy course of muscle fibers with limited areas of spaces in between (arrow B). Fig.12: PTAH stain showing irregular pattern of muscle fibers, limited areas of connective tissue endomysium (brownish color)(arrow A) and variable sized narrow spaces between the muscle fibers (arrow B). Fig.13: H\&E stain in G4B showing keratinized stratified squamous epithelium with acanthotic rete ridges, subepithelial mesenchymal tissue infiltrated by inflammatory cells (arrow A) and abnormal wavy course of muscle fibers with variable sized spaces in between (arrow B). Fig.14: PTAH stain showing irregular pattern of muscle fibers, limited areas of collagen fibers (brownish color) (arrow A) and variable sized narrow spaces between the muscle fibers (arrow B).

\section{Statistical analysis results:}

PTAH staining at 20 days: There was high significant difference between G1 and G2A where $\mathrm{p}$ value was $<0.001$. There was high significant difference between G1 and G3A where p value was $<0.001$. There was high significant difference between G1 and G4A where p value was 0.000. (Fig.15).

PTAH staining at 40 days: There was high significant difference between $\mathrm{G} 1$ and $\mathrm{G} 2 \mathrm{~B}$ where $\mathrm{p}$ value was $<0.001$. There was high significant difference between G1 and G3B where p value was $<0.001$. There was high significant difference between G1 and G4B where p value was 0.000 (Fig.16).

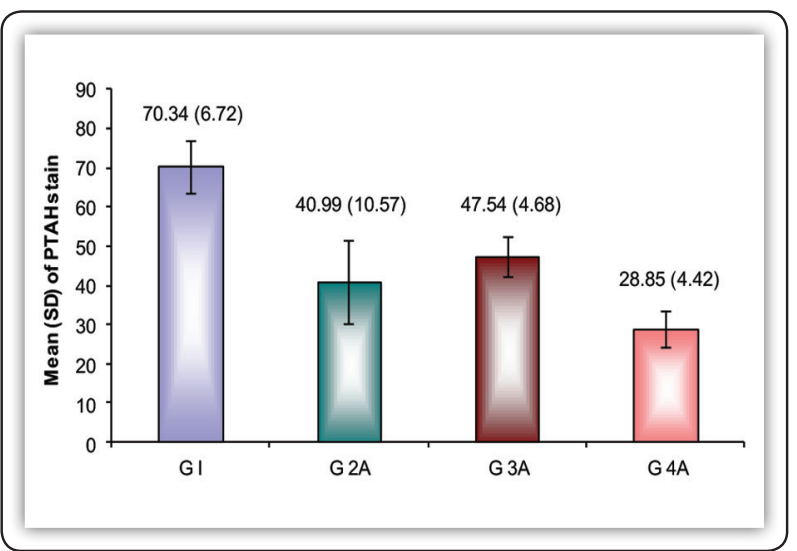

Fig.15: Bar chart representing mean area\% results in all groups at 20 days regarding PTAH stain. 


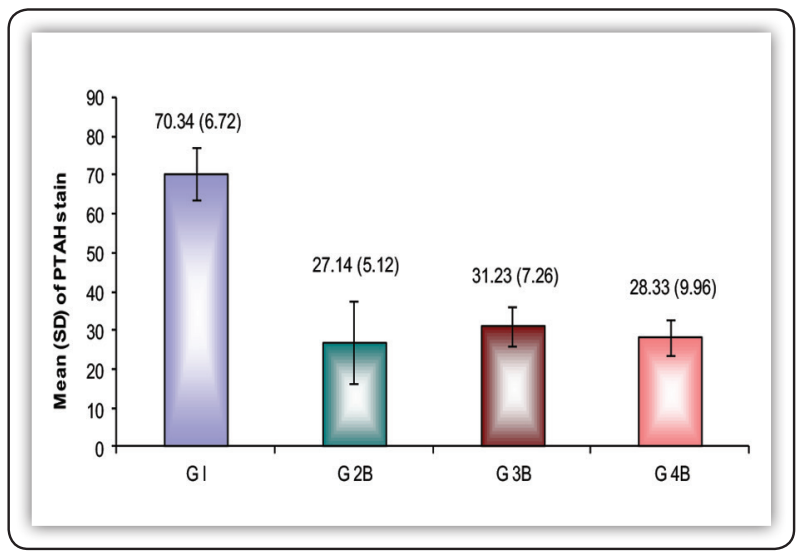

Fig.16: Bar chart representing mean area\% results in all groups at 40 days regarding

\section{DISCUSSION}

Much interest about muscle injury evaluation has been done interchangeably in the literature. Current approaches have been offered systems for classification and grading of muscle injuries. (2) However, a few experimental evidence to support either the pathological or prognostic validity of clinical and histological features during muscle injury as well as when utilizing therapeutic treatment. This in turn made the positive results of the current study are of interest.

To study the process of muscle regeneration in a controlled and reproducible way, it has therefore been necessary to develop animal models of muscle injury ${ }^{(1)}$. The present study showed that adult male albino rats are a successful animal model for the induction of muscle tissue changes. Their muscles, as seen in G1, are similar to those seen by Costa et al. ${ }^{(38)}$ and typically exhibited the same architecture as human one as reported by the aforementioned authors. This in turn provides a useful system upon which to evaluate muscle tissue alterations during injuries as well as when utilizing therapeutic agent. Although the degenerative phase and the regenerative phase of the muscle regeneration process are similar among different muscle types and after varying causes of injuries, the kinetics and amplitude of each phase may vary depending on the extent of the injury, the muscle injured, or the animal model ${ }^{(11)}$.

The use of ACR, in the present study, was due it is considered as one of the major environmental public health problems, results from its increased accumulation in the process of cooking food materials as mentioned on other studies (4). In addition, ACR is a bifunctional monomer containing a reactive electron-deficient double bond and an amide group ${ }^{(11)}$ which is used as a chemical intermediate for the production of polyacrylamides and is identified as a heat-induced process contaminant of carbohydrate rich food ${ }^{(40)}$.

In the current study, serious effects of ACR on gross observation as well as on the histological finding was noticed. The striated muscle fibers of the adult male albino rat's tongue showed variable changes on submicroscopic observation when utilizing either H\&E stain or PTAH stain. The histological features of the tongue mucosa of animals in G2A, those having received ACR for 20 days showed inflammatory cell infiltrates in addition to deteriorations of the pattern muscle fibers with the presence of multiple spaces in-between which reflect a fatty degeneration. Similar results were observed by Weiss et al ${ }^{(41)}$. They noticed that cross-sectional area of myofibers was decreased significantly in association with increased oxidative damage in addition to presence of fatty infiltration, endomysial fibrosis which manifested by increased extracellular matrix between myofibers and these coincides with the result of Al-Serwi et al ${ }^{(19)}$. In the present study, PTAH stain results supported the deterioration of muscle fibers as indicated by area percentage as well as $\mathrm{p}$ value. There were significant differences ( $\mathrm{p} \leq$ 0.001) among the mean area percentage of PTAH staining in G2A compared to G1 (normal group). Muscle regeneration observed in the current study are mostly related to the oxidative stress effect of ACR on the striated muscle. This coincides with the study of Hori et al. ${ }^{(42)}$ and Al-Serwi et al. ${ }^{(19)}$ who 
stated that destruction of muscle fibers with edema and inflammatory cell infiltration are prominent features of oxidative stress effect on skeletal muscle. ACR is a small organic molecule with very high water solubility. These properties facilitate its rapid absorption and distribution through body (35). Various reports suggested that factors released by the injured muscle activate inflammatory cells residing within the muscle, which in turn provide the chemotactic signals to circulating inflammatory cells. Neutrophils are the first inflammatory cells to invade the injured muscle, after neutrophil infiltration and post-injury, macrophages become the predominant inflammatory cell type within the site of injury. Hence oxidative stress has been demonstrated to be a key mechanism in many ACR induced cell injuries and neurodegenerative diseases ${ }^{(43)}$ as well as testicular and epididymal tissues ${ }^{(44)}$. Moreover, ACR toxicity on human and experimental animals has been well documented in a series of reports ${ }^{(19,45)}$. In the current study, the worse effect of ACR was more obvious in gross observation as well as on the histological features in animals of G2B, those having received ACR for 40 days. Splitting of the myofibers and fragmentation of the sarcoplasm in addition to infiltrated by inflammatory cells was shown to be extensive. These results were emphasized by PTAH stain results as indicated by area percentage as well as $p$ value. There were significant differences among the mean area percentage of PTAH in G2B as compared to G1and G2A ( $p \leq 0.001)$. These results are in line with Al-Serwi et al. ${ }^{(19)}$ and Cullen et al. ${ }^{(46)}$ stated that chronic exposure to ACR might lead to skeletal muscle damage in rat tongue which becomes worth with prolonged duration of exposure. Also, Shiono et al. ${ }^{(47)}$ stated that the toxic effects of ACR in different tissues is a dose dependent which increased by increasing the dose or the duration.

The current study reflected the positive effect of thymoquinone as chemopreventive measure on animals in G3A (those having received thymoquinone orally on alternative day with ACR . The gross observations as well as the histological results revealed limited alterations. The tongue mucosa in the animals of G3A 3 animals appeared healthy without observable changes, however, 2 of 5 animals revealed smaller swellings with limited areas of redness compared to that observed in G2(A, B). The histological features reflected this improvement by presence of limited numbers of inflammatory cells. In addition, although some fibers still have wavy course and splitting, limited narrow spaces (fatty degeneration) between muscle fibers and areas of elastic fibers were noticed. The latter reflects the ability of tissue to recoil and return to base line structure after alterations. These results were supported by PTAH stain analysis as indicated by area percentage as well as $\mathrm{p}$ value. There were significant differences among the mean numbers of PTAH in G3A (47.54\%) as compared to G2A $(40.99 \%)$ and $p$ value $(p \leq 0.268)$. These findings may be due to the protective effect of thymoquinone against muscle degeneration. Under acute and chronic pathologic conditions such as ischemia, the balance between oxidant and antioxidant systems has been interrupted ${ }^{(48)}$. Thymoquinone could inhibit non-enzymatic lipid peroxidation in liposomes ${ }^{(49)}$. Also, thymoquinone, has appreciable antioxidant and free radical scavenger properties but no pro-oxidant effect ${ }^{(50)}$. This antioxidant activity may explain the protective effect of this agent against various models in vivo and in vitro ${ }^{(26)}$. and this effect might be be related to the ability of this agent to inhibit lipid peroxidation ${ }^{(51,52) .}$ In the current study, the animals of G3B model, relative its positive effect was noticed when compared to G3A. There were significant differences among the mean numbers of PTAH in G3A (47.54\%) as compared to G3B $(31.23 \%)$ and $p$ value $(\mathrm{p} \leq 0.003)$. The gross observations as well as the histological results revealed some alterations, some areas of redness and swelling in 4 of 5 animals, while the remaining (1 animals) appeared without observable abnormalities. H\&E stain in G3B showed 
keratinized stratified regular acanthotic rete ridges and subepithelial mesenchymal tissue infiltrated by inflammatory cells. Irregular pattern of wavy course muscle fibers with variable sized spaces in between was seen. These results are consistence with those of study ${ }^{(36)}$. This could be due to the effect of thymoquinone administration. Nagi et al. ${ }^{(53)}$ stated that Thymoquinone pretreatment protected various organs against oxidative damage induced by a variety of free radical generating agent which might play an important role as an endogenous antioxidant and could also be applicable as a cytoprotective agent against tissue damage. The results of the present study are in agreement with Nagi et al. ${ }^{(53)}$ statement and supported the potential role of Thymoquinone as a chemopreventive agent in ACR skeletal muscles degeneration. Similarly, Hassan et al. ${ }^{(32)}$ found that the thymoquinone when used as pretreatment during carcinogenesis have stimulated muscle regeneration and expulsion of the inflammatory cells, and resulted in reduced tissue necrosis and faster resolution of the necrotic tissue, i.e. promoting muscle regeneration.

In the current study, the animals of G4A model, were received thymoquinone, as therapeutic measure, intraperitoneally once daily for 3 times as follow: first day, third day and the fifth day. The time choice was because the activated myoblasts accumulate at the site of damage, myoblast proliferation starts around 18-24 h, peaks around 3-4 days, and rapidly declines to cease by about 7-10 days; the precise timing depends on the scale and type of injury ${ }^{(54)}$. The results revealed low positive effect compared to those of G3A\&B. Also, there were significant differences among the mean of the area percentage of PTAH stain in animals in G4A (28.85\%) as compared to those in G3A (47.54) and $p$ value was $(p \leq 0.001)$. These results suggested that massive effect of thymoquinone in chemoprevention rather than treatment of muscle degeneration. The pretreated animals with thymoquinone exhibited higher antioxidant agent contents that their respective controls. Therefore, this agent significantly: increased the antioxidant power of the muscle homogenate samples ${ }^{(36)}$. In the present study, similarly, animals in G4B model revealed it positive effect compared to those of G2A\&B. Contrarily, a relative positive effect was noticed when comparing between G3 and G4. Also, there were non-significant differences among the mean numbers of PTAH stain distribution in G4B (28.33) when compared to G3B (31.23) ( $\mathrm{p} \leq 0.001)$. These results may be related to the fact that regenerative effect of Thymoquinone may require long time of treatment.

\section{CONCLUSION}

A realization of striated muscle damage in rat's tongue by ACR which becomes worth with prolonged duration. Moreover, a significant efficacy of thymoquinone as protective effect was noticed when compared with its therapeutic effect on ACRinduced striated muscle injury of rat's tongue.

\section{REFERENCES}

1. Sophie B, Charge' P, Michael A, Rudnicki. Cellular and molecular regulation of muscle regeneration. Physiol Rev 2004;84(1): 209-38.

2. Hamilton B, Valle X, Rodas G, Til L, Pruna R, Josep et al. Classification and grading of muscle injuries: a narrative review. Br J Sports Med 2015; 49(5):306-13.

3. Rastrollo M, Gortari F, Villegas A, Marti A, Martínez J, González M. A prospective study of eating away-fromhome meals and weight gain in a Mediterranean population: the SUN (Seguimiento Universidad de Navarra) cohort. Public Health Nutr 2010;13(9): 1356-63

4. Losasso C, Cappa V, Neuhouser M, Giaccone V, Andrighetto I, Ricci A. Students' consumption of beverages and snacks at school and away from school: a case study in the north east of Italy. Front Nutr. 2015 2:30- 38

5. EFSA. Scientific opinion on acrylamide in food. EFSA J 2015;13(6):4104-24

6. Haouet N, Pistolese S, Branciari R, Ranucci D, Altissimi M. Study of acrylamide level in food from vending machine. Italian J of Food Safety 2016; 5(4):6147-10. 
7. Hawley G, Lewis R. Hawley's condensed chemical dictionary. Van Nostrand Reinhold;(1997).

8. Exon J. A review of the toxicology of acrylamide. J Toxicol Envi-ron Health B Crit Rev 2006;9(5):397-412.

9. Tareke E, Rydberg P, Karlsson P, Eriksson S, Tornqvist M. Acrylamide: a cooking carcinogen? . Chem Res Toxicol 2000; 13:517-22.

10. Arikawa A, Shiga M. Determination of trace acrylamide in the crops by gas chromatography. Bunseki Kagaku 1980;29(7):33-39.

11. Schultzova J, Tekel J. Acrylamide monomer occurrence in sugar. Dtsch. Lebens 1996; 92:281-82.

12. Friedman M. Chemistry, biochemistry, and safety of acrylamide: a review. J Agric Food Chem 2003;51(16):4504-26.

13. Shipp A, Lawrance G, Gentry R, McDonald T, Bartow H, Bounds J, et al. Acrylamide: review of toxicity data and doseresponse analyses for cancer and non-cancer effects. Crit Rev Toxicol 2006;36(6-7):481-608

14. Zhang Y, Zhang G, Zhang Y. Occurrence and analytical methods of acrylamide in heat-treated foods: review and recent developments. J. Chroma 2005;1075(1-2):1-21.

15. Eriksson S . Acrylamide in food products: identification, formation and analytical methodology. Stockholm, Sweden: Department of Environmental Chemistry, Stockholm University;2005.

16. Parzefall W. Minireview on the toxicity of dietary acrylamide. Food Chem Toxicol 2008;46(4):1360-64.

17. Ma Y, Shi J, Zheng M, Liu J, Tian S, He X, et al. Toxicological effects of acrylamide on the reproductive system of weaning male rats. Toxicol Ind Health 2011;27(7):617-27.

18. Hogervorst J, Baars B, Schouten L, Konings E, Goldbohm R, Brandt P. The carcinogenicity of dietary acrylamide intake: a comparative discussion of epidemiological and experimental animal research. Crit Rev Toxicol 2010;40(6):485-512.

19. Al-Serwia R, Ghoneim F. The impact of vitamin E against acrylamide induced toxicity on skeletal muscles of adult male albino rat tongue: Light and electron microscopic study. J Micros Ultrastruc 2015;3(3): 137-47.

20. Prasad S. Evidence of acrylamide induced oxidative stress and neurotoxicity in Drosophila melanogaster: its ameliora-tion with spice active enrichment: relevance to neuropathy. Neurotoxicology 2012;33(5):1254-64.

21. Canton M, Menazza S, Di Lisa F, "Oxidative stress in muscular dystrophy: from generic evidence to specific sources and targets". J Muscle Res and Cell Motility 2014;35(1):23-36.

22. Choi M, Ow J, Yang M, Taneja R. Oxidative stress-mediated skeletal muscle degeneration: molecules, mechanisms, and therapies. Oxid Med Cell Longev 2016; 2016:1-13.

23. Halliwell B, "Biochemistry of oxidative stress," Biochem Soc Trans. 2007;35(5):1147-50.

24. Brand M. "The sites and topology of mitochondrial superoxide production," Exp Gerontol 2010;45(7-8): 466-72.

25. Relaix F, Zammit P. Satellite cells are essential for skeletal muscle regeneration: the cell on the edge returns center stage. Development 2012;139(16):2845-56.

26. Tamaki T, Uchiyama Y, Akatsuka A. Plasticity and physiological role of stem cells derived from skeletal muscle interstitium: Contribution to muscle fiber hyperplasia and therapeutic use. Curr Pharm Des 2010; 16(8): 956-67.

27. Robertson T, Grounds M, Mitchell C, Papadimitriou J. Fusion between myogenic cells in vivo: An ultra-structural study in regenerating murine skeletal muscle. J Struc Biol 1990; 105(1-3):170-82.

28. Robertson T, Papadimitriou J, \& Grounds M. Fusion of myogenic cells to the newly sealed region of damaged myofibrils in skeletal muscle regeneration. Neuropatho Appl Neurobio 1993; 19: 350-58.

29. Wieteska w, Grzelkowska k, Rejmak E. factor and cytokine interactions in myogenesis. Part II. Expression of IGF binding proteins and protein kinases essential for myogenesis in mouse $\mathrm{C} 2 \mathrm{C} 12$ myogenic cells exposed to TNFalpha and IFN-gamma. Pol J Vet Sci 2011a; 14(3):425-31.

30. Wieteska W, Grzelkowska K, Tokarska J, Grabiec K. Growth factor and cytokine interactions in myogenesis. Part I. The effect of TNF-alpha and IFN-gamma on IGFI-dependent differentiation in mouse $\mathrm{C} 2 \mathrm{C} 12$ myogenic cells. Pol J Vet Sci 2011b; 14(3):417-24.

31. Hosseinzadeh H, Modaghegh M, Saffari Z. Crocus sativus L. (saffron) extract and its active constituents (crocin and safranal) ischemia-reperfusion in rat skeletal muscle. Evid Based Complement Alternat Med 2009; 6(3):343-50.

32. Hassan M, Abdel-Latif G, El-Hossary W. Protective and promising myogenic effect of two thymoquinone formulations in relation to the hamster buccal pouch-induced carcinogenic Model IOSR J2017; 16(5):54-66.

33. Al-Saleh I, Billedo G, El-Doush. Levels of selenium, DLa-tocopherol, DLc- tocopherol, all-trans-retinol, thymoquinone and thymol in different brands of Nigella sativa seeds J Food Compos Anal 2006;19(2-3): 167 -75. 
34. Pereira S, Tettamanti M: Ahimsa and alternatives. The concept of the 4th R. The CPCSEA in India.Altex 2005; 22(1): 3-6.

35. Lopachin R, Balaban C, Ross J. Acrylamide axonopathy revisited. toxico appl pharmacol. 2003; 188(3):135-53.

36. Hosseinzadeh H, Taiari S, Nassiri-Asl M. Effect of thymoquinone, a constituent of Nigella sativa L., on ischemiareperfusion in rat skeletal muscle. Naunyn Schmiedebergs Arch Pharmacol 2012;385(5):503-8.

37. Horiuchi N, Aihara N, Mizutani H, Kousaka S, Nagafuchi $\mathrm{T}$, Ochiai M, et al. Becker muscular dystrophy-like myopathy regarded as so-called "fatty muscular dystrophy" in a Pig: a case report and its diagnostic method. J Vet Med Sci 2014;76(2):243-48

38. Costa A, Pereira C, Junqueira J, Jorge A. Recent mouse and rat methods for the study of experimental oral candidiasis. Virulence 2013;4(5):391-99.

39. Pavlath G, Thaloor D, Rando T, Cheong M, English A, Zheng B. Heterogeneity among muscle precursor cells in adult skeletal muscles with differing regenerative capacities. Dev Dyn 1998; 212(4): 495-508.

40. JECFA. Evaluation of certain contaminants in food. Joint FAO/WHO expert committee on food additives, Rome, Italy 2011.

41. Weiss D, Casale G, Koutakis P, Nella A, Swanson S, Zhu $\mathrm{Z}$, et al. Oxidative damage and myofiber degeneration in the gastrocnemius of patients with peripheral arterial disease. J Trans 1Med 2013;11: 230-39.

42. Hori K, Tsujii M, Iino T, Satonaka H, Uemura T, AkedaK, et al. Protective effect of edaravone for tourniquet-induced ischemia-reperfusion injury on skeletal muscle in murine hindlimb. BMC Musculoskelet Disord 2013; 14:113.

43. Ali M, Aly E, Elawady A. Effectiveness of selenium on acryl-amide toxicity to retina. Int J Ophthalmol 2014; 7(4):614-20.

44. Lebda M, Gad S, Gaafar H. Effects of lipoic acid on acrylamide induced testicular damage. Mater Sociomed 2014;26(3):208-12.
45. Raju J, Sondagar C, Roberts J, Aziz S, Caldwell D, Vavasour E et al. Dietary acrylamide does not increase colon aberrantcrypt foci formation. Food Chem Toxicol 2011;49(6):1373-80.

46. Cullen M, Johnson M, Mastaglia F. Pathological reactions of skeletal muscle. In: Mastaglia FL, Walton JN, editors. Skeletal muscle pathology. Edinburgh: Churchill Livingstone; 2001 123-84.

47. Shinomol G, Raghunath N, Bharath M, Muralidhara. Prophylaxis with Bacopa monnieri attenuates acrylamide induced neurotoxicity and oxidative damage via elevated antioxidant function. Cent Nerv Syst Agents Med Chem 2013;13(1):3-12.

48. Abdollahi M, Ranjbar R, Shadnia S, Nikfar S, Rezaie A Pesticides and oxidative stress: a review. Med Sci Mon 2004; 10(6):141 -7

49. Abdel-Fattah A, Matsumoto K, Watanabe H. Antinociceptive effects of Nigella sativa oil and its major component, thymoquinone, in mice. Eur J Pharmacol 2000;400(1):89-97.

50. Ismail $\mathrm{M}, \mathrm{Al}-\mathrm{Naqeep} \mathrm{G}, \mathrm{Chan} \mathrm{K}$. Nigella sativa thymoquinone rich fraction greatly improves plasma antioxidant capacity and expression of antioxidant genes in hypercholesterolemic rats. Free Radic Biol Med 2010;48(5):664-72.

51. Al-Gharably N, Badary O, Nagi M, Al-Sawaf H, Al Rikabi A, Al-Bekairi A. Protective effect of thymoquinone against carbon tetrachloride-induced hepatotoxicity in mice. Res Commun Pharmacol Toxicol 1997;2(1): 41- 50.

52. Worthen D, Ghosheh O, Crooks P. The in vitro anti-tumor activity of some crude and purified components of blackseed Nigella sativa L. Anticancer Res 1998; 18(3):1527-32.

53. Nagi M, Alam K, Badary O, Al-Shabaneh O, Al-Sawaf H, Al- Bekairi A. Thymoquinone protects against carbon tetrachloride hepatotoxicity in mice via an antioxidant mechanism. Biochem Mol Biol Int 1999 ;47(1):153-9.

54. Mc Geachie, J, Grounds M. Initiation and duration of muscle precursor replication after mild and severe injury to skeletal muscle of mice. An auto radiographic study. Cell Tis Res 1987; 248(1), 125-30. 